\title{
Coarse Aperture Study \\ for the \\ Window Frame Dipoles in RHIC
}

Armando Anti11ón

BNL, August 3, 1984

\section{ABSTRACT}

We use PATRICIA to study the quality of the multipolar contribution in the Window Frame Dipoles (WFD). In order to avoid possible noise with insertions, we only use 81 characteristic RHIC cell. As we want to make a comparison with available information on the Dedicated RHIC dipoles (DRD), we only determine the aperture of WFD for some values of $\Delta p / p$. 
This exercise is made in order to provide information, from the dynamical point of view, of possible magnets to be used in RHIC. This study has already been made for the dedicated RHIC dipoles ${ }^{1}$, and we will take it as reference to compare the WFD results. The structure of the cells are of the FODO type with a dipole (10.7m) in between. Two families of sextupoles (with zero length) have been used to correct the natural chromaticityto a zero value. One family is in the center of the focusing quadrupole and the other in the center of the defocusing quadrupole. The multipolar contribution to ideal dipoles is simulated with zero length multipoles in the edges of dipoles. This is a rough approach, and work to take a better integration along dipoles is under progress.

Usua11y people in magnet design use coefficients ( $b_{n}^{\prime}$ ) that give directly the deviation of the field, respect $B$, in a certain reference point, i.e.

$$
\frac{B}{B_{0}}=\sum_{n} b_{n}^{\prime}\left(\frac{x}{x_{0}}\right)^{n} \quad \text {, where } x_{0} \text { is the reference point, }
$$

while Patricia use as input coefficients bn given by

$$
\frac{B}{B_{0}}=\sum_{n} b_{n} x^{n}
$$

The obvious relation between them is

$$
b_{n}=\frac{b_{n}^{\prime}}{x_{0}^{n}}
$$

The coefficients $b_{n}^{\prime}$ have been provided by J.Jackson, and for low field dipoles $(<1.9 \mathrm{~T})$ they are as follows 6thers which are not shown are zero for $\mathrm{n}<22$ )

\begin{tabular}{|r|c|c|}
\hline $\mathrm{n}$ & bn $\times 10^{4}($ at $2.54 \mathrm{~cm})$ & $\mathrm{bn}$ \\
\hline 4 & -.08 & -19.2201 \\
6 & .08 & $2.9791 \times 10^{4}$ \\
8 & .02 & $1.15441 \times 10^{7}$ \\
18 & .01 & $5.16408 \times 10^{22}$ \\
20 & -.01 & $-8.00434 \times 10^{25}$ \\
\hline
\end{tabular}

The quality of the dipole field is measured making a comparision between maximun beam size for ideal magriets (no multipoles) and maximum beam size whèn multipoles are included. The beam size in one direction is determined with the emittance and the $\beta$ function as $U(s)=\sqrt{\frac{\epsilon_{u} \beta_{u}(s)}{\pi}}$

For a given aperture of the elements in the ring, we count the number of Eurns that a particle can give in the ring without exceding in amplitude the aperture imposed. This will give information about the physical aperture. WThen 
this physical barrier is made too large $(1000 \mathrm{~mm})$, we will have information :about the dynamical stability of the beam, i.e. the dynamical aperture.

We chose in PATRICIA a reference emittance of $\epsilon_{0}=0.5 \pi \times 10^{-6} \mathrm{k}-\mathrm{rad}$ in terms of its units, emittance is controlled by a parameter $A$ in the way

$$
\epsilon=\left(\frac{A}{10}\right)^{2} \epsilon_{0}
$$

Changing $A$ we can see the number of turns a particle can give in the ring.

1.- Dynamical Aperture of Dipoles.

We will present results in the same way as in 1 ).

A has the same meaning as before.

$B$ is the number of turns the particle will give if its amplitude does not exceed the aperture given.

$C$ is the number of turns the particle gives in the ring.

$\mathrm{D}=$ beam size $=\left(\mathrm{x}^{2}+\mathrm{y}^{2}\right)^{\frac{3}{2}}$. With $\epsilon=\epsilon_{x}=\epsilon_{y}, \quad D=\left[\frac{\epsilon}{\pi}\left(\beta_{x}+\beta_{y}\right)\right]^{\frac{1}{2}}$ At the end of dipoles $\quad \beta x+\beta y \sim 52.7 m$

\begin{tabular}{|l|r|l|}
\hline$\frac{\Delta P}{P}(\%)$ & $A(B)$ & $D(\mathrm{~mm})$ \\
\hline+0.2 & $80(100)$ & $>41.0$ \\
0.0 & $115(100)$ & $>59.0$ \\
-0.2 & $80(100)$ & $>41.0$ \\
\hline
\end{tabular}

Table I.- Dipoles with no multipoles have larger dynamical aperture than that shown (taken from reference 1)

\begin{tabular}{|c|c|c|}
\hline$\Delta P / P(\%)$ & $A(B), A(C)$ & $D(m)$ \\
\hline+0.2 & $99(100), 101(62)$ & $50.8 \leq \mathrm{D} \leq 51.8$ \\
0.0 & $109(100), 111(93)$ & $56.0 \leq \mathrm{D} \leqslant 56.9$ \\
-0.2 & $107(100), 109(100)$ & $54.9 \leq \mathrm{D} \leqslant 56.0$ \\
\hline
\end{tabular}

Table II.- Dipoles with Window Frame multipoles. Dynamical aperture.

Last table shows that at $\frac{A P}{P}=0$ the dynamical aperture of WFD is $\sim 20 \mathrm{~mm}$ bigger than the corresponding DRD aperture.

2.- Physical Aperture of Dipoles.

To determine how stable is the beam near the operation emittance, a physi- 
cal aperture of $30 \mathrm{~mm}$ has been taken in the elements of the lattice. Next table shows these results.

\begin{tabular}{|c|c|c|}
\hline$\Delta P / P(\%)$ & $A(B), A(C)$ & $D(m m)$ \\
\hline+0.2 & $48(100), 50(6)$ & $24.6 \leq D \leq 25.7$ \\
0.0 & $50(100), 52(13)$ & $25.7 \leq D \leq 26.7$ \\
-0.2 & $44(100), 46(25)$ & $22.6 \leq D \leq 23.6$ \\
\hline
\end{tabular}

Table III.- Dipoles with Window Frame multipoles. Physical aperture.

If we consider that the physical aperture for ideal dipoles (without multipoles) is of the same magnitude as for $D R D,(26.7 \leqslant D \leqslant 27.7)$, then for the WFD, the physical aperture is $\sim 1 \mathrm{~mm}$ smaller. Finally we must to point out that this is not the real physical aperture. In order to determine it is necesary, as we already mentioned, to integrate the effects of multipoles in smaller pieces in each dipole.

\section{References.}

1.- G.F. Del1, Aperture Dependence of RHIC cell on multipole fields in the Dedicated RHIC Dipoles. Internal Report.

2.- J. Jackson, Private conmunication. 
In this annex we will show the dynamical and physical apertures when we split the dipoles in three pieces, as we see in the next figure:

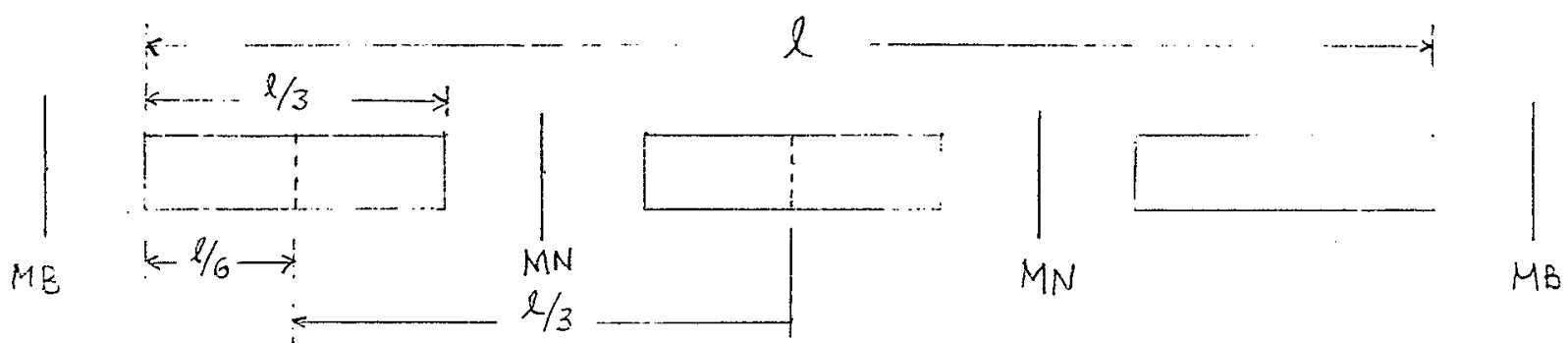

where $\ell=10.7 \mathrm{~m}, M B$ and $M N$ have lengths $\ell / 6$ and $l / 3$ respectively. The program PATRICIA has been changed ${ }^{3}$ to avoid the focusing forces at the edges of the dipole where $M N$ is.

The tune, for which the aperture studies were made, is shown in the following tune diagram

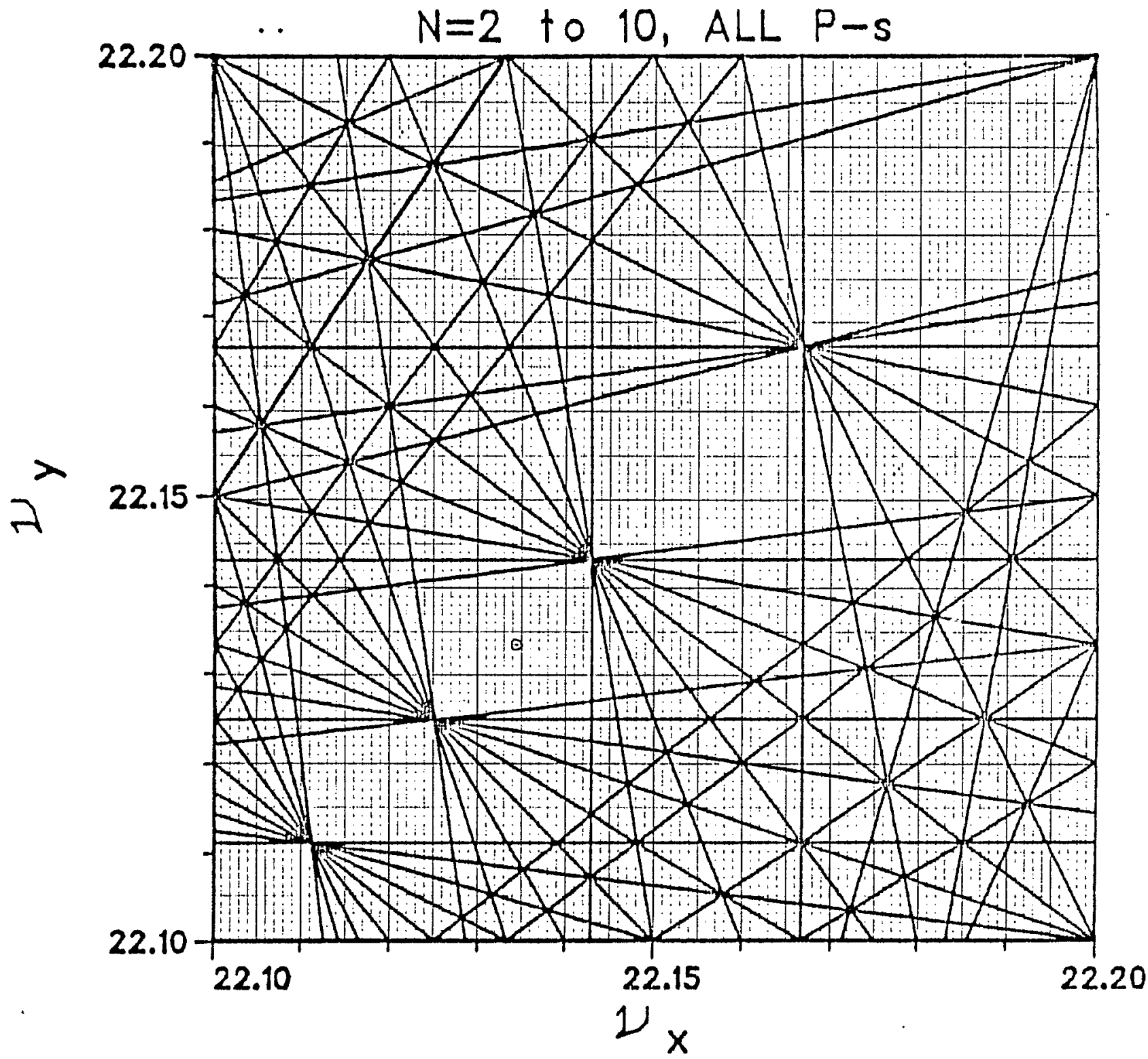


The dynamical aperture is now:

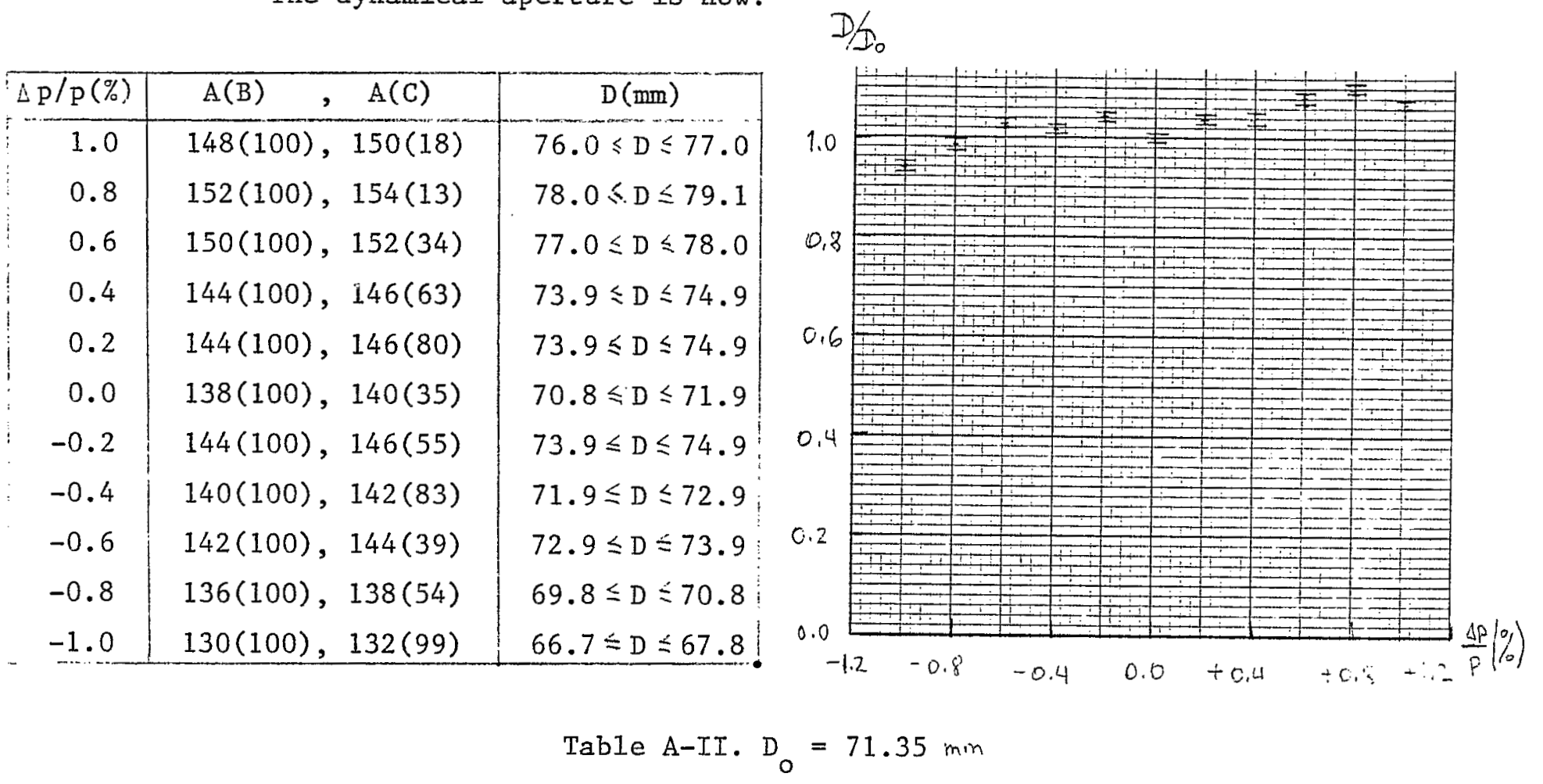

and the physical aperture is:

\begin{tabular}{|c|c|c|}
\hline$\Delta p / p(\%)$ & , $A(C)$ & $D(\mathrm{~mm})$ \\
\hline 1.0 & $27(100), 29(17)$ & $13.9 \leqslant D \leqslant 14.9$ \\
\hline 0.8 & $35(100), 36(1)$ & $18.0 \leq D \leq 18.5$ \\
\hline 0.6 & $40(100), 42(1)$ & $20.5 \leq D \leq 21.6$ \\
\hline 0.4 & $44(100), 46(1)$ & $22.6 \leq D \leq 23.6$ \\
\hline 0.2 & $48(100), 50(5)$ & $24.6 \leq D \leq 25.7$ \\
\hline 0.0 & $50(100), 52(19)$ & $25.7 \leqslant D \leqslant 26.7$ \\
\hline-0.2 & $46(100), 48(14)$ & $23.6 \leqslant D \leqslant 24.6$ \\
\hline-0.4 & $40(100), 42(29)$ & $20.5 \leqslant D \leqslant 21.6$ \\
\hline-0.6 & $34(100), 36(44)$ & $17.5 \leqslant D \leqslant 18.5$ \\
\hline-0.8 & $27(100), 29(73)$ & $13.9 \leq D \leq 14.9$ \\
\hline-1.0 & $23(100), 25(65)$ & $11.8 \leq \mathrm{D} \leqslant 12.8$ \\
\hline
\end{tabular}

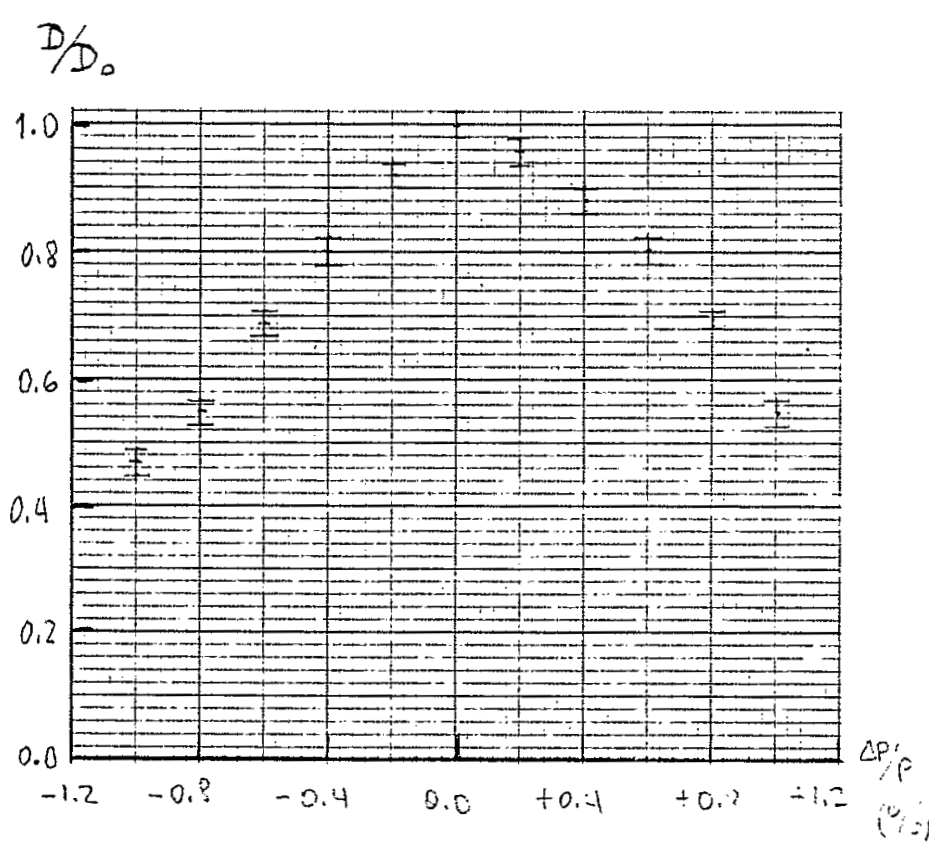

Table A-III. $D_{0}=26.2$ min 